\title{
High Hematocrit Levels Are Associated with Risk of Cardiovascular Mortality among Middle-Aged Japanese Women: The Ibaraki Prefectural Health Study (IPHS)
}

\author{
Nobuko Takaoka, ${ }^{1,2,3}$ Toshimi Sairenchi, ${ }^{1,3,4}$ Fujiko Irie, ${ }^{5}$ Munehiro Matsushita,,${ }^{1,6}$ \\ Masanori Nagao, ${ }^{7}$ Mitsumasa Umesawa, ${ }^{3,8}$ Yasuo Haruyama, ${ }^{1}$ Hiroshi Watanabe, ${ }^{4}$ \\ Kazumasa Yamagishi, ${ }^{3,4,9}$ Hiroyasu Iso, ${ }^{10}$ Gen Kobashi $^{1}$ and Hitoshi Ota ${ }^{3,4}$

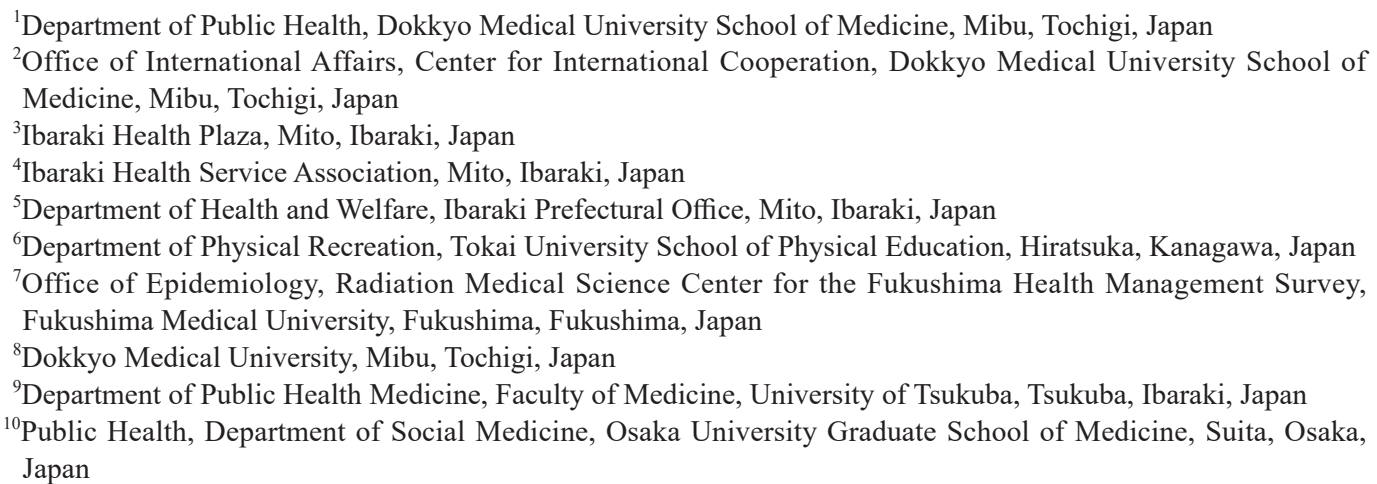

In Japan, heart disease and cerebrovascular disease were the second and fourth leading cause of death, respectively in 2014. Hematocrit, the ratio of the red blood cells to the total volume of blood is known to play a role in cardiovascular diseases. However, the relationship between elevated hematocrit and the risk of cardiovascular disease based on sex has not been examined in Asian countries. We analyzed data from the Ibaraki Prefectural Health Study, a community-based large cohort study, which included 87,533 individuals, aged 40 to 79 years living in 38 municipalities of the Ibaraki Prefecture, who had completed an annual health checkup in 1993, and were followed until 2013. The hematocrit levels of the subjects were categorized into 5 quintiles (Q1-Q5), and hazard ratios for cause-specific mortality were calculated using the Cox proportional hazards regression models. Age and other cardiovascular risk factors were the covariates in the study. During the follow-up for a mean of 17.9 years, 1,207 deaths (615 men and 592 women) due to acute myocardial infarction (AMI) were reported in this population. The $p$ values for the trend in the Q3-Q5 groups were 0.661 for men and 0.020 for women. Additionally, these values were significant in younger (40 to 59 years) women but not in older (60 to 79 years) women. This study is the first one to demonstrate an association between high hematocrit level and risk of AMI mortality in younger Japanese women, but not in men and older women.

Keywords: acute myocardial infarction; cardiovascular diseases; cohort studies; hematocrit; Japanese women Tohoku J. Exp. Med., 2019 September, 249 (1), 65-73. (C) 2019 Tohoku University Medical Press

\section{Introduction}

Cardiovascular mortality accounts for 25\%-60\% of deaths in industrialized countries, such as the United States, Germany, Italy, and the Russian Federation (World Health Organization 2014). In Japan, heart disease and cerebrovascular disease were the second and fourth leading cause of death respectively in 2014 (Ministry of Health, Labour and Welfare 2016). Therefore, prevention of cardiovascular disease (CVD) is important in reducing the overall mortality.

Hematocrit, ratio of the volume of red blood cells to the volume of whole blood, is a major determinant of blood viscosity, blood pressure, venous return, cardiac output, and platelet adhesiveness (Guyton and Richardson 1961; Hellem et al. 1961; Lowe et al. 1985; Vazquez et al. 2010). Thus, high hematocrit level could burden the cardiovascular system. The possible mechanism of the independent asso-

Received August 5, 2019; revised and accepted September 18, 2019. Published online September 28, 2019; doi: 10.1620/tjem.249.65.

Correspondence: Nobuko Takaoka, Department of Public Health, Dokkyo Medical University School of Medicine, 880 Kita-kobayashi, Mibu, Tochigi 321-0293, Japan. e-mail: takaoka@dokkyomed.ac.jp 
ciation between high hematocrit level and acute myocardial infarction (AMI) mortality is unclear. However, evidence shows that hemorheologic factors may play an important role in the development of cardiovascular events (Nicolaides et al. 1977; Thomas et al. 1977; Tohgi et al. 1978; Lowe et al. 1980, Gotoh et al. 2015). Given the correlation between the hematocrit and thrombotic risk (Eischer et al. 2012), studies tend to focus on hematocrit level rather than hemoglobin level.

Previous epidemiological studies have reported that high hematocrit level can be a risk factor for CVD (Tohgi et al. 1978; Carter et al. 1983; Kiyohara et al. 1986; Gotoh et al. 2015). Studies from the United States have shown this association in women, but not in men (Gagnon et al. 1994; Brown et al. 2001). The Framingham Heart Study (Gagnon et al. 1994) found that, for CVD mortality, an increased multivariable-adjusted odds ratio of the highest versus intermediate quintiles of hematocrit level was seen in older (65 to 94 years old) men and younger (35 to 64 years old) women. Meanwhile, coronary heart disease (CHD) mortality, showed a significant association with high hematocrit only in younger women, but not in men. The Second National Health and Nutrition Examination Survey (NHANES II) indicated no association between hematocrit in the upper versus lower tertile, and mortality from either CHD, disease of heart or CVD among men. Women with hematocrit in the upper tertile, were 1.3 times (95\% CI, 0.91.9) more likely to die from CHD than those in the lowest tertile (Brown et al. 2001).

In the general Japanese population, the association of high hematocrit level with the risk of CVD mortality, stratified by sex is still not clear. In 2015, Japan had the lowest rate $(3.7 \%)$ of obesity in among adults compared to the United States (38.2\%) and other in member countries of the Organization for Economic Co-operation and Development (OECD 2017) (19.5\%). In this study, we analyzed data from the Ibaraki Prefectural Health Study (IPHS), the largest Japanese cohort study to examine whether high hematocrit level could be a risk factor for cardiovascular mortality, stratified by sex and age in a population, which has the lowest obesity rate.

\section{Methods}

\section{Study population}

The IPHS, a community-based large cohort study, was conducted by the Ibaraki prefectural government to obtain data on the health status of the residents for the purpose of health education and policy making (Sairenchi et al. 2011). There were 85 municipalities in the Ibaraki Prefecture in 1993. The cohort included 97,078 individuals (33,138 men and 63,940 women) aged 40 to 79 years living in 38 of the 85 municipalities who had completed an annual health checkup in 1993, and were followed up until 2013.

Of these, 4,506 individuals with incomplete health checkup data and 5,039 with a history of stroke or heart disease at baseline were excluded from the study. Finally, we analyzed data from 87,533 individuals $(29,781$ men and 57,752 women) in the present study.
Informed consent was obtained from community representatives to conduct the epidemiological study. The Ethics Committee of the Ibaraki Prefecture and the Bioethics Committee of Dokkyo Medical University approved this study.

\section{Baseline measurements}

Blood samples were drawn from subjects in a seating position. Fasting was not needed, hematocrit was measured automatically (Beckman Coulter S-PLUS and STKR Analyzer, Beckman Coulter Inc., Tokyo, Japan) using the impedance method. Other biochemical parameters used in the analyses were measured as previously reported (Irie et al. 2006; Sairenchi et al. 2011; Nagai et al. 2016).

\section{Follow-up Surveillance}

The incidence of death in the cohort was determined by systematically reviewing the death certificates. The details regarding the methods for surveillance have been reported previously (Irie et al. 2006; Sairenchi et al. 2011; Nagai et al. 2016).

The underlying causes of death in this cohort were obtained from the Ministry of Health, Labor and Welfare database, where they had been coded for the National Vital Statistics according to the International Classification of Diseases (ICD), 9th (1993-1994) and 10th (1995-2013) revisions.

\section{Definitions}

We categorized the hematocrit levels into 5 quintiles, with 1 (Q1) being the lowest and 5 (Q5) being the highest. Smoking habits were divided into 4 categories as follows: never smoker, ex-smoker, currently smoking $<20$ cigarettes/d, and currently smoking $\geq 20$ cigarettes/d. Alcohol intake was categorized as never, sometimes (not almost every day), almost every day $(<44 \mathrm{~g} / \mathrm{d})$, and almost every day $(\geq 44 \mathrm{~g} / \mathrm{d})$.

We classified cause-specific mortality according to the ICD code of the underlying cause of death. AMI deaths were identified as code 410 in the ICD-9 and as code I21 in the ICD-10. Deaths due to strokes, cerebral infarction, intracerebral hemorrhage, and subarachnoid hemorrhage were coded as reported previously (Sairenchi et al. 2011)

\section{Statistical analysis}

The $\mathrm{p}$ values for differences in the baseline characteristics of individuals categorized into the 5 hematocrit levels were calculated using the analysis of variance (ANOVA). These characteristics included age, body mass index (BMI), systolic blood pressure, diastolic blood pressure, serum total cholesterol level, serum high-density lipoprotein cholesterol level, and serum creatinine level. The chisquare test was used to analyze data on the use of antihypertensive, antidyslipidemic, and antidiabetic medications, proteinuria, atrial fibrillation, ST-T abnormality, left ventricular hypertrophy, KeithWagener-Barker Classification, smoking status, and alcohol intake.

Hazard ratios and $95 \%$ confidence intervals (CIs) for all-cause and cause-specific mortality were calculated with reference to the intermediate quintile (Q3) using the Cox proportional hazards regression models. Q3 was chosen as a reference because previous studies have described a U-shaped association between hematocrit and mortality (Gagnon et al. 1994; Boffetta et al. 2013). The covariates for analysis included age (continuous), BMI, systolic blood pressure, use of antihypertensive medications (yes or no), serum total cholesterol level, serum high-density lipoprotein cholesterol level, use of anti- 
dyslipidemic medications (yes or no), blood glucose status (normal, prediabetes, and diabetes mellitus), use of antidiabetic medication (yes or no), serum creatinine level, proteinuria (yes or no), atrial fibrillation (yes or no), ST-T abnormality (yes or no), left ventricular hypertrophy (yes or no), smoking status and alcohol intake, and the Keith-Wagener-Barker Classification (normal, grade 1, grade 2, grade 3). The Keith-Wagener-Barker is criteria which classify hypertensive retinopathy. According to the Keith-Wagener-Barker classification (Keith et al. 1974), grade 1 is defined as mild narrowing or sclerosis of the retinal arterioles. Grade 2 is defined as moderate to marked sclerosis of the retinal arterioles, moderate narrowing of the retinal arterioles, or arteriosclerotic retinopathy or thrombosis of retinal veins. Grade 3 is defined as angiospastic retinopathy, characterized by edema, cotton-wool patches, and hemorrhages in the retina, in addition to marked sclerosis of the retinal arterioles.

The $\mathrm{p}$ values for trend among Q3-Q5 of hematocrit level were also calculated via multivariate Cox proportional hazard models.

All statistical tests were 2 sided, and values of $p<0.05$ were considered statistically significant. All statistical analyses were conducted with SAS version 9.4 (SAS Institute, Inc., Cary, NC).

\section{Results}

Tables 1 and 2 summarize the baseline characteristics

Table 1. Baseline characteristics according to the quintile of hematocrit levels for men.

\begin{tabular}{|c|c|c|c|c|c|c|}
\hline Hematocrit (Quintile) & Q1 & Q2 & Q3 & Q4 & Q5 & $\begin{array}{c}\mathrm{p} \text { for } \\
\text { Difference }^{*}\end{array}$ \\
\hline \multicolumn{7}{|l|}{ Men } \\
\hline Participants, $n$ & 6,047 & 5,807 & 5,995 & 6,059 & 5,873 & \\
\hline Hematocrit (min-max) & $17.3-41.5$ & $41.6-43.5$ & $43.6-45.2$ & $45.3-47.2$ & $47.3-64.3$ & \\
\hline Hematocrit (median) & 39.9 & 42.6 & 44.4 & 46.2 & 48.7 & \\
\hline Age, y & $64.9(8.5)$ & $61.3(9.5)$ & $59.7(9.8)$ & $58.0(10.0)$ & $56.4(10.0)$ & $<0.001$ \\
\hline Body mass index, $\mathrm{kg} / \mathrm{m}^{2}$ & $21.9(2.8)$ & $22.9(2.8)$ & $23.4(2.8)$ & $23.9(2.8)$ & $24.5(2.9)$ & $<0.001$ \\
\hline Systolic blood pressure, $\mathrm{mm} \mathrm{Hg}$ & $135.8(17.8)$ & $136.0(17.3)$ & $135.7(17.2)$ & $136.2(17.4)$ & $137.7(17.5)$ & $<0.001$ \\
\hline Diastolic blood pressure, $\mathrm{mm} \mathrm{Hg}$ & $78.5(10.6)$ & $80.0(10.3)$ & $80.8(10.4)$ & $81.8(10.7)$ & $83.9(10.8)$ & $<0.001$ \\
\hline Use of antihypertensive medications, $\%$ & 21.3 & 18.8 & 19.0 & 18.7 & 19.4 & 0.0017 \\
\hline Serum total cholesterol level, $\mathrm{mmol} / \mathrm{L}$ & $4.7(0.8)$ & $4.9(0.8)$ & $5.0(0.8)$ & $5.1(0.9)$ & $5.2(0.9)$ & $<0.001$ \\
\hline $\begin{array}{l}\text { Serum high-density lipoprotein } \\
\text { cholesterol level, mmol/L }\end{array}$ & $1.4(0.4)$ & $1.4(0.4)$ & $1.4(0.4)$ & $1.3(0.4)$ & $1.3(0.4)$ & $<0.001$ \\
\hline $\begin{array}{l}\text { Use of antidyslipidemic } \\
\text { medications, \% }\end{array}$ & 1.0 & 1.1 & 1.3 & 1.2 & 1.4 & 0.302 \\
\hline Diabetes mellitus, $\%$ & 5.3 & 4.5 & 5.0 & 4.9 & 7.7 & $<0.001$ \\
\hline Use of antidiabetic medications , $\%$ & 3.9 & 3.3 & 3.4 & 3.5 & 4.0 & 0.145 \\
\hline Serum creatinine level, $\mathrm{mmol} / \mathrm{L}$ & $90.5(16.4)$ & $88.6(13.3)$ & $89.0(13.0)$ & $89.2(12.3)$ & $90.4(12.6)$ & $<0.001$ \\
\hline Proteinuria, $\%$ & 3.2 & 2.5 & 2.5 & 2.7 & 5.0 & $<0.001$ \\
\hline Atrial fibrillation, $\%$ & 0.5 & 0.6 & 0.5 & 0.6 & 1.0 & 0.0018 \\
\hline ST-T abnormality, $\%$ & 1.8 & 1.4 & 1.7 & 1.7 & 1.9 & 0.434 \\
\hline \multicolumn{7}{|l|}{$\begin{array}{l}\text { Retinal photography } \\
\text { (Keith-Wagener-Barker } \\
\text { Classification), } \%\end{array}$} \\
\hline Normal & 69.8 & 74.5 & 75.8 & 77.8 & 77.3 & $<0.001$ \\
\hline Grade 1 & 23.9 & 21.1 & 20.5 & 17.8 & 18.3 & \\
\hline Grade 2 & 6.1 & 4.3 & 3.6 & 4.2 & 4.4 & \\
\hline Grade 3 & 0.2 & 0.1 & 0.1 & 0.1 & 0.0 & \\
\hline \multicolumn{7}{|l|}{ Smoking status, $\%$} \\
\hline Never smoker & 23.9 & 23.5 & 23.6 & 21.3 & 18.3 & $<0.001$ \\
\hline Ex-smoker & 28.8 & 27.9 & 27.4 & 25.8 & 22.6 & \\
\hline $\begin{array}{l}\text { Currently smoking }<20 \\
\text { cigarettes } / \mathrm{d}\end{array}$ & 19.2 & 16.1 & 14.6 & 13.5 & 13.2 & \\
\hline $\begin{array}{l}\text { Currently smoking } \geq 20 \\
\text { cigarettes/d }\end{array}$ & 28.1 & 32.5 & 34.5 & 39.4 & 45.9 & \\
\hline \multicolumn{7}{|l|}{ Alcohol intake, $\%$} \\
\hline Never & 43.0 & 34.4 & 33.0 & 31.1 & 27.8 & $<0.001$ \\
\hline Sometimes & 12.9 & 13.9 & 13.9 & 14.2 & 14.1 & \\
\hline Almost every day $<44 \mathrm{~g} / \mathrm{d}$ & 39.5 & 45.1 & 46.0 & 47.0 & 47.7 & \\
\hline Almost every day $\geq 44 \mathrm{~g} / \mathrm{d}$ & 4.6 & 6.5 & 7.2 & 7.8 & 10.4 & \\
\hline
\end{tabular}

For men: Q1, 17.3-41.5; Q2, 41.6-43.5; Q3, 43.6-45.2; Q4, 45.3-47.2; Q5,47.3-64.3.

*Age, body mass index, systolic blood pressure, diastolic blood pressure, serum total cholesterol level, serum highdensity lipoprotein cholesterol level, and serum creatinine level were calculated via ANOVA, while use of antihypertensive, antidyslipidemic, and antidiabetic medications, diabetes mellitus, proteinuria, atrial fibrillation, ST-T abnormality, left ventricular hypertrophy, Keith-Wagener-Barker Classification, smoking status, and alcohol intake were calculated via $\mathrm{x}^{2}$ test. Values are presented as mean (SD) when appropriate. 
Table 2. Baseline characteristics according to the quintile of hematocrit levels for women.

\begin{tabular}{|c|c|c|c|c|c|c|}
\hline Hematocrit (Quintile) & Q1 & Q2 & Q3 & Q4 & Q5 & $\begin{array}{c}\mathrm{p} \text { for } \\
\text { Difference }^{*}\end{array}$ \\
\hline \multicolumn{7}{|l|}{ Women } \\
\hline Participants, $\mathrm{n}$ & 11,415 & 11,925 & 11,073 & 11,859 & 11,480 & \\
\hline Hematocrit (min-max) & $13.7-36.7$ & $36.8-38.6$ & $38.7-40.0$ & $40.1-41.7$ & $41.8-53.8$ & \\
\hline Hematocrit (median) & 35.2 & 37.8 & 39.4 & 40.8 & 43.0 & \\
\hline Age, y & $57.0(11.3)$ & $58.0(10.2)$ & $57.5(10.0)$ & $57.3(9.7)$ & $57.8(9.5)$ & $<0.001$ \\
\hline Body mass index, $\mathrm{kg} / \mathrm{m}^{2}$ & $22.7(3.0)$ & $23.2(3.1)$ & $23.6(3.1)$ & $23.9(3.2)$ & $24.6(3.3)$ & $<0.001$ \\
\hline Systolic blood pressure, $\mathrm{mm} \mathrm{Hg}$ & $128.6(17.7)$ & $130.2(17.5)$ & $131.3(17.6)$ & $132.6(17.7)$ & $135.8(17.9)$ & $<0.001$ \\
\hline Diastolic blood pressure, $\mathrm{mm} \mathrm{Hg}$ & $74.9(10.2)$ & $76.5(10.3)$ & $77.6(10.3)$ & $78.7(10.2)$ & $81.1(10.6)$ & $<0.001$ \\
\hline Use of antihypertensive medications, $\%$ & 14.1 & 17.4 & 18.3 & 20.0 & 26.0 & $<0.001$ \\
\hline Serum total cholesterol level, $\mathrm{mmol} / \mathrm{L}$ & $5.1(0.8)$ & $5.3(0.9)$ & $5.4(0.9)$ & $5.5(0.9)$ & $5.6(0.9)$ & $<0.001$ \\
\hline $\begin{array}{l}\text { Serum high-density lipoprotein } \\
\text { cholesterol level, mmol/L }\end{array}$ & $1.5(0.4)$ & $1.5(0.4)$ & $1.5(0.4)$ & $1.5(0.4)$ & $1.5(0.4)$ & 0.002 \\
\hline Use of antidyslipidemic medications, $\%$ & 2.0 & 3.0 & 3.3 & 3.3 & 4.2 & $<0.001$ \\
\hline Diabetes mellitus, $\%$ & 1.3 & 1.5 & 2.0 & 2.9 & 5.3 & $<0.001$ \\
\hline Use of antidiabetic medications, $\%$ & 1.2 & 1.5 & 1.9 & 2.2 & 3.6 & $<0.001$ \\
\hline Serum creatinine level, $\mathrm{mmol} / \mathrm{L}$ & $69.8(14.0)$ & $69.7(11.3)$ & $69.6(11.1)$ & $69.8(10.8)$ & $70.3(11.1)$ & $<0.001$ \\
\hline Proteinuria, \% & 1.8 & 1.4 & 1.5 & 1.5 & 2.4 & $<0.001$ \\
\hline Atrial fibrillation, $\%$ & 0.1 & 0.1 & 0.1 & 0.1 & 0.2 & 0.0138 \\
\hline ST-T abnormality, $\%$ & 1.8 & 1.8 & 2.1 & 2.3 & 3.0 & $<0.001$ \\
\hline Left ventricular hypertrophy, $\%$ & 2.2 & 1.8 & 1.4 & 1.3 & 1.3 & $<0.001$ \\
\hline \multicolumn{7}{|l|}{$\begin{array}{l}\text { Retinal photography } \\
\text { (Keith-Wagener-Barker } \\
\text { Classification), \% }\end{array}$} \\
\hline Normal & 80.9 & 79.2 & 79.1 & 78.7 & 76.8 & $<0.001$ \\
\hline Grade 1 & 15.5 & 17.1 & 17.1 & 17.4 & 18.7 & \\
\hline Grade 2 & 3.5 & 3.7 & 3.6 & 3.9 & 4.4 & \\
\hline Grade 3 & 0.1 & 0.1 & 0.1 & 0.1 & 0.1 & \\
\hline \multicolumn{7}{|l|}{ Smoking status, $\%$} \\
\hline Never smoker & 96.0 & 95.7 & 95.1 & 94.1 & 91.2 & $<0.001$ \\
\hline Ex-smoker & 0.6 & 0.6 & 0.7 & 0.8 & 0.9 & \\
\hline Currently smoking $<20$ cigarettes $/ \mathrm{d}$ & 2.6 & 2.6 & 2.9 & 3.3 & 4.5 & \\
\hline Currently smoking $\geq 20$ cigarettes $/ \mathrm{d}$ & 0.8 & 1.1 & 1.3 & 1.8 & 3.5 & \\
\hline \multicolumn{7}{|l|}{ Alcohol intake, \% } \\
\hline Never & 91.9 & 91.0 & 90.1 & 89.6 & 88.9 & $<0.001$ \\
\hline Sometimes & 5.6 & 5.7 & 6.3 & 6.5 & 6.7 & \\
\hline Almost every day $<44 \mathrm{~g} / \mathrm{d}$ & 2.4 & 3.3 & 3.5 & 3.7 & 4.2 & \\
\hline Almost every day $\geq 44 \mathrm{~g} / \mathrm{d}$ & 0.1 & 0.1 & 0.1 & 0.1 & 0.2 & \\
\hline
\end{tabular}

For women: Q1, 13.7-36.7; Q2, 36.8-38.6; Q3, 38.7-40.0; Q4, 40.1-41.7; Q5, 41.8-53.8.

*Age, body mass index, systolic blood pressure, diastolic blood pressure, serum total cholesterol level, serum high-density lipoprotein cholesterol level, and serum creatinine level were calculated via ANOVA, while use of antihypertensive medication, antidyslipidemic, and antidiabetic medications, diabetes mellitus, proteinuria, atrial fibrillation, ST-T abnormality, left ventricular hypertrophy, Keith-Wagener-Barker Classification, smoking status, and alcohol intake were calculated via $\mathrm{x}^{2}$ test. Values are presented as mean (SD) when appropriate.

for men and women on the basis of their hematocrit levels. Statistically significant differences according to these 5 categories were found for all covariables at baseline, except for the use of antidyslipidemic medications, antidiabetic medications use, and ST-T abnormality in men.

During follow-up through 2013, for a mean of 17.9 years (17.0 years in men and 18.3 years in women), 1,207 AMI deaths (615 men and 592 women), 2,716 total stroke deaths (1,219 men and 1,497 women), 1,612 cerebral infarction deaths (800 men and 812 women), 668 intracerebral hemorrhage deaths (297 men and 371 women), and 380 subarachnoid hemorrhage deaths (98 men and 282 women) were observed.
Table 3 shows the hazard ratios of AMI mortality based on the hematocrit levels. The multivariable hazard ratio for the highest quintile was marginally significantly higher in women, but not in men. The $\mathrm{p}$ value for the trend among intermediate to highest hematocrit levels (Q3-Q5) was significant in women $(\mathrm{p}=0.020)$, but not in men $(\mathrm{p}=$ $0.661)$. Meanwhile, by age group, the $\mathrm{p}$ value for the trend among intermediate to highest hematocrit levels (Q3-Q5) was significant in younger (40 to 59$)$ women $(\mathrm{p}=0.028)$, but not in older (60 to 79) women ( $\mathrm{p}=0.103$ ) (Table 4$)$.

Table 5 shows the multivariable hazard ratios for stroke and stroke type mortality based on the quintiles of hematocrit levels among all study subjects. No significant 
Table 3. Hazard ratios of acute myocardial infarction mortality according to the quintile of hematocrit levels.

\begin{tabular}{|c|c|c|c|c|c|c|c|c|c|}
\hline & & Person years & No. of deaths & \multicolumn{2}{|c|}{ Age-adjusted HR } & $\mathrm{p}$ & \multicolumn{2}{|c|}{ Multivariable-adjusted $\mathrm{HR}^{\dagger}$} & $\mathrm{p}$ \\
\hline \multirow[t]{6}{*}{ Men } & All & $507,056.7$ & 615 & & & & & & \\
\hline & Q1 & $93,365.4$ & 160 & 1.17 & $(0.91-1.49)$ & 0.215 & 1.21 & $(0.94-1.56)$ & 0.133 \\
\hline & Q2 & $98,531.9$ & 118 & 1.02 & $(0.79-1.32)$ & 0.886 & 1.05 & $(0.81-1.36)$ & 0.718 \\
\hline & Q3 & $104,292.0$ & 109 & 1 & (reference) & & 1 & (reference) & \\
\hline & Q4 & $107,080.3$ & 115 & 1.17 & $(0.90-1.52)$ & 0.254 & 1.11 & $(0.86-1.45)$ & 0.430 \\
\hline & Q5 & $103,787.1$ & 113 & 1.35 & $(1.04-1.76)$ & 0.025 & 1.16 & $(0.89-1.52)$ & 0.269 \\
\hline \multicolumn{2}{|c|}{$\mathrm{p}$ for trend $\mathrm{d}^{*}$} & & & 0.033 & & & 0.661 & & \\
\hline \multirow[t]{6}{*}{ Women } & All & $1,058,018.3$ & 592 & & & & & & \\
\hline & Q1 & $206,196.1$ & 121 & 1.14 & $(0.87-1.49)$ & 0.333 & 1.20 & $(0.92-1.57)$ & 0.184 \\
\hline & Q2 & $218,685.1$ & 120 & 1.06 & $(0.81-1.39)$ & 0.663 & 1.09 & $(0.84-1.42)$ & 0.528 \\
\hline & Q3 & $204,421.3$ & 99 & 1 & (reference) & & 1 & (reference) & \\
\hline & Q4 & $218,990.3$ & 113 & 1.13 & $(0.86-1.47)$ & 0.391 & 1.09 & $(0.83-1.43)$ & 0.517 \\
\hline & Q5 & $209,725.5$ & 139 & 1.43 & $(1.10-1.84)$ & 0.007 & 1.26 & $(0.97-1.64)$ & 0.084 \\
\hline \multicolumn{2}{|c|}{$\mathrm{p}$ for trend $\mathrm{d}^{*}$} & & & $<0.001$ & & & 0.020 & & \\
\hline
\end{tabular}

For men: Q1, 17.3-41.5; Q2, 41.6-43.5; Q3, 43.6-45.2; Q4, 45.3-47.2; Q5, 47.3-64.3.

For women: Q1, 13.7-36.7; Q2, 36.8-38.6; Q3, 38.7-40.0; Q4, 40.1-41.7; Q5, 41.8-53.8.

HR, hazard ratio.

${ }^{\dagger}$ Adjusted for age, body mass index systolic blood pressure, use of antihypertensive medication (yes or no), serum total cholesterol level, serum high-density lipoprotein cholesterol level, use of antidyslipidemic medication (yes or no), glycometabolic status (normal, prediabetes, and diabetes mellitus), use of antidiabetic medication (yes or no), serum creatinine level, proteinuria (yes or no), atrial fibrillation (yes or no), ST-T abnormality (yes or no), left ventricular hypertrophy (yes or no), Keith-Wagener-Barker Classification (normal, grade 1 , grade 2, and grade 3), smoking status (never smoker, ex-smoker, currently smoking $<20$ cigarettes/d, currently smoking $\geq 20$ cigarettes/d), and alcohol intake (never, sometimes, $<44 \mathrm{~g} / \mathrm{d}$ almost every day, and $\geq 44 \mathrm{~g} / \mathrm{d}$ almost every day).

* $\mathrm{p}$ for trend: Calculated for subjects of Q3-Q5.

associations were found between hematocrit levels and risks of mortality from stroke or stroke types.

\section{Discussion}

To the best of our knowledge, this is the first study to show the association between high hematocrit levels and the risk of AMI mortality in a general population of Japanese women, but not men.

Several studies have analyzed the associations between high hematocrit level and CVD, CHD, and stroke. While six studies (Sorlie et al. 1981; Carter et al. 1983; Gagnon et al. 1994; Kunnas et al. 2009; Boffetta et al. 2013; Gotoh et al. 2015) found significant associations between high hematocrit levels and the incidence as well as mortality related to CVD, CHD, or MI, one study (Brown et al. 2001) found no such associations. Of these, the Framingham Heart Study (Gagnon et al. 1994) investigated the association between high hematocrit level and CVD mortality with a 34-year follow-up, and reported differences in the effect of high hematocrit based on sex and age. It also found that for CVD mortality, an increased multivariable-adjusted odds ratio of the highest versus intermediate quintile of hematocrit level was seen in older (65 to 94 years old, $p=0.05$ ) men and younger (35 to 64 years old, $\mathrm{p}=0.02$ ) women. Meanwhile, no significant association was seen with the incidence of MI. The Golestan Cohort Study in Iran (Boffetta et al. 2013) investigated the association between high hematocrit and CVD mortality for a mean follow-up period of 5 years. Values were categorized into units of $5 \%$, except for the lowest and highest categories. The study found that, for the CVD mortality in women, the adjusted hazard ratio for the highest hematocrit group compared to the third-lowest hematocrit group was 2.87 (95\% CI, 1.495.52). In men, the adjusted hazard ratio in the highest hematocrit group compared to the intermediate hematocrit group was $1.70(95 \% \mathrm{CI}, 0.75-3.85)$. The study of the Japanese-American men in Hawaii (Honolulu Heart Program) (Carter et al. 1983), the Framingham Heart Study (Gagnon et al. 1994), and the Tampere adult population cardiovascular risk study (TAMRISK) (Kunnas et al. 2009) in Finland found significant associations between high hematocrit levels and CHD mortality, while the NHANES II Mortality Study (Brown et al. 2001) did not find any significant association.

The Hisayama Study (Gotoh et al. 2015) found that using the third quartile of hematocrit as a reference, the highest quartiles significantly increased the multivariable risk ratio for the incidence of CVD and CHD, in men and women combined. Our analysis based on sex revealed high hematocrit level might be an independent risk factor of AMI mortality among women.

A Puerto Rican cohort study (Sorlie et al. 1981) found that the incidence of death related to MI and CHD was more than double in the high hematocrit group. However, 
N. Takaoka et al.

Table 4. Hazard ratios of acute myocardial mortality according to the quintile of hematocrit levels by age group.

\begin{tabular}{|c|c|c|c|c|c|c|c|c|c|}
\hline \multicolumn{2}{|c|}{ Age group } & $\begin{array}{l}\text { Person } \\
\text { Years }\end{array}$ & $\begin{array}{l}\text { Deaths } \\
\mathrm{n}\end{array}$ & \multirow[t]{2}{*}{ AAHR } & \multirow[t]{2}{*}{$95 \% \mathrm{CI}$} & \multirow[t]{2}{*}{$\mathrm{p}$} & \multirow[t]{2}{*}{$\mathrm{MAHR}^{\dagger}$} & \multirow[t]{2}{*}{$95 \% \mathrm{CI}$} & \multirow[t]{2}{*}{$\mathrm{p}$} \\
\hline \multicolumn{4}{|l|}{$\overline{\text { Men }}$} & & & & & & \\
\hline & Total & $507,056.7$ & 615 & & & & & & \\
\hline $40-59$ & All & $230,872.7$ & 91 & & & & & & \\
\hline & Q1 & $45,370.4$ & 17 & 1.13 & $(0.55-2.29)$ & 0.745 & 1.28 & $(0.62-2.63)$ & 0.509 \\
\hline & Q2 & $45,251.4$ & 16 & 1.16 & $(0.57-2.38)$ & 0.687 & 1.27 & $(0.62-2.61)$ & 0.513 \\
\hline & Q3 & $48,896.8$ & 14 & 1 & (reference) & & 1 & (reference) & \\
\hline & Q4 & $45,463.7$ & 16 & 1.31 & $(0.64-2.69)$ & 0.461 & 1.26 & $(0.61-2.58)$ & 0.535 \\
\hline & Q5 & $45,890.4$ & 28 & 2.29 & $(1.20-4.35)$ & 0.012 & 1.88 & $(0.98-3.63)$ & 0.058 \\
\hline $\mathrm{p}$ for $\mathrm{tr}$ & end ${ }^{*}$ & & & 0.009 & & & 0.238 & & \\
\hline $60-79$ & All & $276,184.0$ & 524 & & & & & & \\
\hline & Q1 & $50,679.1$ & 109 & 1.10 & $(0.84-1.43)$ & 0.512 & 1.09 & $(0.83-1.43)$ & 0.547 \\
\hline & $\mathrm{Q} 2$ & $53,431.9$ & 95 & 0.96 & $(0.73-1.27)$ & 0.079 & 0.97 & $(0.73-1.28)$ & 0.801 \\
\hline & Q3 & $57,863.8$ & 104 & 1 & (reference) & & 1 & (reference) & \\
\hline & Q4 & $57,391.3$ & 104 & 1.06 & $(0.81-1.39)$ & 0.671 & 1.00 & $(0.76-1.31)$ & 0.985 \\
\hline & Q5 & $56,817.9$ & 112 & 1.19 & $(0.91-1.56)$ & 0.200 & 1.04 & $(0.79-1.37)$ & 0.787 \\
\hline $\mathrm{p}$ for $\mathrm{tr}$ & $e^{*}$ & & & 0.198 & & & 0.494 & & \\
\hline \multicolumn{10}{|l|}{ Women } \\
\hline & Total & $1,058,018.3$ & 592 & & & & & & \\
\hline $40-59$ & All & $592,553.9$ & 60 & & & & & & \\
\hline & Q1 & $118,633.1$ & 10 & 1.47 & $(0.59-3.62)$ & 0.407 & 1.66 & $(0.67-4.14)$ & 0.278 \\
\hline & Q2 & $118,356.2$ & 8 & 0.94 & $(0.36-2.43)$ & 0.892 & 1.01 & $(0.39-2.62)$ & 0.987 \\
\hline & Q3 & $122,652.1$ & 9 & 1 & (reference) & & 1 & (reference) & \\
\hline & Q4 & $116,362.4$ & 10 & 1.17 & $(0.47-2.87)$ & 0.736 & 1.08 & $(0.44-2.67)$ & 0.866 \\
\hline & Q5 & $116,550.1$ & 23 & 2.56 & $(1.19-5.54)$ & 0.017 & 1.88 & $(0.85-4.16)$ & 0.118 \\
\hline $\mathrm{p}$ for $\mathrm{tr}$ & end ${ }^{*}$ & & & 0.009 & & & 0.028 & & \\
\hline $60-79$ & All & $465,464.4$ & 532 & & & & & & \\
\hline & Q1 & $87,563.0$ & 111 & 1.11 & $(0.84-1.46)$ & 0.462 & 1.15 & $(0.87-1.52)$ & 0.330 \\
\hline & Q2 & $94,222.2$ & 105 & 1.06 & $(0.81-1.40)$ & 0.658 & 1.09 & $(0.83-1.44)$ & 0.549 \\
\hline & Q3 & $95,951.4$ & 97 & 1 & (reference) & & 1 & (reference) & \\
\hline & Q4 & $94,552.4$ & 103 & 1.13 & (0.86-1.49) & 0.394 & 1.10 & (0.83-1.46) & 0.494 \\
\hline & Q5 & $93,175.4$ & 116 & 1.31 & $(1.00-1.72)$ & 0.049 & 1.19 & $(0.90-1.56)$ & 0.218 \\
\hline $\mathrm{p}$ for $\mathrm{tr}$ & end ${ }^{*}$ & & & 0.047 & & & 0.103 & & \\
\hline
\end{tabular}

\footnotetext{
For men: Q1, 17.3-42.8; Q2, 40.8-44.5; Q3, 42.8-46.1; Q4, 44.6-47.9; Q5, 46.6-64.3.

For women: Q1, 13.7-36.7; Q2, 36.8-38.6; Q3, 38.6-40.1; Q4, 40.1-41.7; Q5, 41.8-53.8.

AAHR, age-adjusted hazard ratio; MAHR, multivariable adjusted hazard ratio; CI, confidence interval.

†Adjusted for age, body mass index, systolic blood pressure, use of antihypertensive medications (yes or no), serum total cholesterol level, serum high-density lipoprotein cholesterol level, use of antidyslipidemic medications (yes or no), glycometabolic status (normal, prediabetes, and diabetes mellitus), use of antidiabetic medications (yes or no), serum creatinine level, proteinuria (yes or no), atrial fibrillation (yes or no), ST-T abnormality (yes or no), left ventricular hypertrophy (yes or no), Keith-Wagener-Barker Classification (normal, grade 1, grade 2, and grade 3), smoking status (never smoker, ex-smoker, currently smoking $<20$ cigarettes/d, currently smoking $\geq 20$ cigarettes/d), and alcohol intake (never, sometimes, $<44 \mathrm{~g} / \mathrm{d}$ almost every day, and $\geq 44 \mathrm{~g} / \mathrm{d}$ almost every day).

*p for trend: Calculated for subjects of Q3-Q5.
}

three studies (Gagnon et al. 1994; Brown et al. 2001; Gotoh et al. 2015) have reported varying effects of high hematocrit based on sex and different age groups.

Concurrently, some studies (Beltrame et al. 1999; Pristipino et al. 2000) indicated that differences in coronary artery vasomotor reactivity may exist among races. In addition to the racial differences, a pathological study on MI reported differences between Japanese men in rural (Akita) and urban (Osaka) areas (Konishi et al. 1992). While massive-type infarction was less common in the rural area compared to the urban area, the opposite trend was seen for scattered-type infarction (Konishi et al. 1992). The Ni-Hon-San (Nippon, Honolulu, San Francisco) project showed that old cerebral infarcts and intraparenchymal artery sclerosis of the Japanese subjects (Hiroshima) were significantly more frequent than the Honolulu subjects (Mitsuyama et al. 1979). This study indicates that differences in race and lifestyle could cause inconsistent findings.

Our study examined whether high hematocrit level could be a risk factor for cardiovascular mortality, stratified by sex in a general Japanese population. The results indicated that multivariable hazard ratio of AMI for high hema- 
Table 5. Multivariable hazard ratios of cause-specific mortality according to the quintile of hematocrit levels.

\begin{tabular}{|c|c|c|c|c|c|c|}
\hline & Hematocrit (Quintile) & Q1 & Q2 & Q3 & Q4 & Q5 \\
\hline \multicolumn{7}{|l|}{ Men } \\
\hline & Person-years & $93,365.4$ & $98,531.9$ & $104,292.0$ & $107,080.3$ & $103,787.1$ \\
\hline & \multicolumn{6}{|l|}{ Stroke } \\
\hline & Deaths,n & 368 & 246 & 242 & 196 & 167 \\
\hline & Multivariable $\mathrm{HR}^{\dagger}$ & 1.10 & 0.93 & 1 & 0.90 & 0.89 \\
\hline & $95 \% \mathrm{CI}$ & $0.93-1.30$ & $0.78-1.11$ & reference & $0.75-1.09$ & $0.73-1.09$ \\
\hline & \multicolumn{6}{|l|}{ Cerebral infarction } \\
\hline & Deaths,n & 239 & 163 & 165 & 133 & 100 \\
\hline & Multivariable $\mathrm{HR}^{\dagger}$ & 1.00 & 0.89 & 1 & 0.92 & 0.81 \\
\hline & $95 \% \mathrm{CI}$ & $0.82-1.22$ & $0.72-1.11$ & reference & $0.73-1.15$ & $0.63-1.04$ \\
\hline & \multicolumn{6}{|l|}{ Cerebral hemorrhage } \\
\hline & Deaths,n & 90 & 57 & 52 & 47 & 51 \\
\hline & Multivariable $\mathrm{HR}^{\dagger}$ & 1.28 & 1.00 & 1 & 0.99 & 1.25 \\
\hline & $95 \% \mathrm{CI}$ & $0.90-1.82$ & $0.68-1.46$ & reference & $0.67-1.47$ & $0.85-1.86$ \\
\hline & \multicolumn{6}{|l|}{ Subarachnoid hemorrhage } \\
\hline & Deaths,n & 32 & 22 & 19 & 10 & 15 \\
\hline & Multivariable $\mathrm{HR}^{\dagger}$ & 1.57 & 1.17 & 1 & 0.53 & 0.82 \\
\hline & $95 \% \mathrm{CI}$ & $0.87-2.84$ & $0.63-2.16$ & reference & $0.25-1.15$ & $0.41-1.64$ \\
\hline \multicolumn{7}{|c|}{ Women } \\
\hline & Person-years & $206,196.1$ & $218,685.1$ & $204,421.3$ & $218,990.3$ & $209,725.5$ \\
\hline & \multicolumn{6}{|l|}{ Stroke } \\
\hline & Deaths,n & 385 & 280 & 261 & 283 & 288 \\
\hline & Multivariable $\mathrm{HR}^{\dagger}$ & 1.37 & 0.94 & 1 & 1.04 & 1.03 \\
\hline & $95 \% \mathrm{CI}$ & $1.17-1.61$ & $0.80-1.12$ & reference & $0.87-1.23$ & $0.87-1.23$ \\
\hline & \multicolumn{6}{|l|}{ Cerebral infarction } \\
\hline & Deaths,n & 198 & 150 & 136 & 161 & 167 \\
\hline & Multivariable $\mathrm{HR}^{\dagger}$ & 1.31 & 0.95 & 1 & 1.14 & 1.16 \\
\hline & $95 \% \mathrm{CI}$ & $1.05-1.64$ & $0.76-1.20$ & reference & $0.90-1.43$ & $0.92-1.46$ \\
\hline & \multicolumn{6}{|l|}{ Cerebral hemorrhage } \\
\hline & Deaths,n & 111 & 68 & 67 & 67 & 58 \\
\hline & Multivariable $\mathrm{HR}^{\dagger}$ & 1.54 & 0.90 & 1 & 0.96 & 0.81 \\
\hline & $95 \% \mathrm{CI}$ & $1.13-2.09$ & $0.64-1.26$ & reference & $0.68-1.35$ & $0.57-1.16$ \\
\hline & \multicolumn{6}{|l|}{ Subarachnoid hemorrhage } \\
\hline & Deaths,n & 71 & 56 & 54 & 49 & 52 \\
\hline & Multivariable $\mathrm{HR}^{\dagger}$ & 1.31 & 0.95 & 1 & 0.86 & 0.89 \\
\hline & $95 \% \mathrm{CI}$ & $0.92-1.88$ & $0.65-1.38$ & reference & $0.58-1.26$ & $0.60-1.30$ \\
\hline
\end{tabular}

For men: Q1,17.3-41.5; Q2, 41.6-43.5; Q3, 43.6-45.2; Q4, 45.3-47.2; Q5, 47.3-64.3.

For women: Q1, 13.7-36.7; Q2, 36.8-38.6; Q3, 38.7-40.0; Q4, 40.1-41.7; Q5, 41.8-53.8.

$\mathrm{HR}$, hazard ratio; $\mathrm{CI}$, confidence interval.

${ }^{\dagger}$ Adjusted for age, body mass index, systolic blood pressure, use of antihypertensive medications (yes or no), serum total cholesterol level, serum high-density lipoprotein cholesterol level, use of antidyslipidemic medications (yes or no), glycometabolic status (normal, prediabetes, and diabetes mellitus), use of antidiabetic medications (yes or no), serum creatinine level, proteinuria (yes or no), atrial fibrillation (yes or no), ST-T abnormality (yes or no), left ventricular hypertrophy (yes or no), Keith-Wagener-Barker Classification (normal, grade 1, grade 2, and grade 3), smoking status (never smoker, ex-smoker, currently smoking $<20$ cigarettes/d, currently smoking $\geq 20$ cigarettes/d), and alcohol intake (never, sometimes, $<44 \mathrm{~g} / \mathrm{d}$ almost every day, and $\geq 44 \mathrm{~g} / \mathrm{d}$ almost every day).

tocrit was marginally significant in women, but not in men, though the mechanism underlying this association is unclear. However, several studies show that hemorheologic factors may play an important role in the development of cardiovascular events (Nicolaides et al. 1977; Thomas et al. 1977; Tohgi et al. 1978; Lowe et al. 1980; Gotoh et al. 2015). Hematocrit, the proportion of blood volume by red blood cell, is a major determinant of blood viscosity and may have serious effects on organ perfusion. The associa- tion of hematocrit with other hematologic factors, such as platelets or fibrinogen, may influence the development of cardiovascular events (Harrison et al. 1984; Lowe and Forbes 1985; Lowe 1998; Lowe et al. 2002). An Austrian cohort study (Eischer et al. 2012) found a strong association between hematocrit and the risk of recurrent venous thrombosis (VTE) in women, but not in men. In the current study, we found a significant association between high hematocrit level and AMI mortality only in younger 
women. The mechanism by which sex and age affect the association between hematocrit level and AMI is unknown. The sex hormone estrogen is known to increase arterial vasodilation, inhibit the cellular response to vascular injury, as well as prevents atherosclerosis, menopause and the formation of coronary artery plaques, all of which might be the influential factors. The Framingham study found that the risk factors for CVD significantly increased in the fifth decade of women's life, which is 10 years later than that in men (Kannel et al. 1976). Several studies have suggested that the onset of menopause is an influential factor for the varying results based on age and sex (Kannel et al. 1976; Skafar et al. 1997; Mendelsohn and Karas 1999; BarrettConnor 2013). Furthermore, a review suggested that the direct action of estrogen on blood vessels provides a cardiovascular protective effect (Mendelsohn and Karas 1999). Therefore, during menopause (45 to 55 years of age), the decrease in estrogen levels could lead to increased risk for cardiac events. Another study indicated a difference in the composition of the coronary artery plaques between men and women (Davies 1997). As the group of younger women in our study comprised of those aged from 40 to 59 years, menopause transition and differences in plaque composition might have produced varying results based on sex and age.

The strength of the present study is the use of a longitudinal large population-based cohort for sex-stratified and cause-specific analysis. In addition, all blood samples were measured using the same devices, reagents, and quality control programs. This study has some limitations. First, while several studies have indicated circadian and seasonal variations in the hematocrit (Touitou et al. 1986; Kubota et al. 1987; Pocock et al. 1989; Sennels et al. 2011), we could not adjust for these variations because the health checkups were conducted throughout the year. Second, information on the history and status of menstruation, gynecological disorders, and treatment for anemia was not obtained.

In conclusion, high hematocrit levels are associated with risk of mortality from AMI among younger Japanese women, but not men and older women.

\section{Acknowledgments}

This work was supported by a Grant-in-Aid from the Ministry of Health, Labor and Welfare, Health and Labor Sciences Research Grants, Japan (Research on Health Services: H17-Kenkou-007; Comprehensive Research on Cardiovascular and Life-Style Related Diseases: H18-Junkankitou[Seishuu]Ippan-012; Comprehensive Research on Cardiovascular and Life-Style Related Diseases: H20-Junkankitou[Seishuu]-Ippan013; Intractable Diseases Conquest Research: H21-NanchiIppan-059; Comprehensive Research on Cardiovascular and Life-Style Related Diseases: H23-Junkankitou[Seishuu]-Ippan005; an Intramural Research Fund (22-4-5) for Cardiovascular Diseases of National Cerebral and Cardiovascular Center; Comprehensive Research on Cardiovascular and Life-StyleRelated Diseases: H26-Junkankitou [Seisaku]-Ippan-001; and Comprehensive Research on Cardiovascular and Life-StyleRelated Diseases: H29-Junkankitou [Seishuu]-Ippan-003).

\section{Author Contributions}

Nobuko Takaoka participated in the design of the study, analyzed the data, and wrote the first draft and the final report with all authors contributing to the editing. Toshimi Sairenchi, Fujiko Irie and Hitoshi Ota participated in the design of the study. Toshimi Sairenchi, Gen Kobashi, Hiroyasu Iso, and Kazumasa Yamagishi gave important suggestions for the design of this study and interpretation of the results. Fujiko Irie and Hirosi Watanabe arranged the acquisition of individual data. Masanori Nagao, Mitsumasa Umesawa, Yasuo Haruyama, and All authors gave important suggestions for writing the draft and revising it critically for important intellectual content, and read and approved the final manuscript.

\section{Conflict of Interest}

The authors declare no conflict of interest.

\section{References}

Barrett-Connor, E. (2013) Menopause, atherosclerosis, and coronary artery disease. Curr. Opin. Pharmacol., 13, 186-191.

Beltrame, J.F., Sasayama, S. \& Maseri, A. (1999) Racial heterogeneity in coronary artery vasomotor reactivity: differences between Japanese and Caucasian patients. J. Am. Coll. Cardiol., 33, 1442-1452.

Boffetta, P., Islami, F., Vedanthan, R., Pourshams, A., Kamangar, F., Khademi, H., Etemadi, A., Salahi, R., Semnani, S., Emadi, A., Abnet, C.C., Brennan, P., Pharoah, P.D., Dawsey, S.M. \& Malekzadeh, R. (2013) A U-shaped relationship between haematocrit and mortality in a large prospective cohort study. Int. J. Epidemiol., 42, 601-615.

Brown, D.W., Giles, W.H. \& Croft, J.B. (2001) Hematocrit and the risk of coronary heart disease mortality. Am. Heart J., 142, 657-663.

Carter, C., McGee, D., Reed, D., Yano, K. \& Stemmermann, G. (1983) Hematocrit and the risk of coronary heart disease: the Honolulu Heart Program. Am. Heart J., 105, 674-679.

Davies, M.J. (1997) The composition of coronary-artery plaques. N. Engl. J. Med., 336, 1312-1314.

Eischer, L., Tscholl, V., Heinze, G., Traby, L., Kyrle, P.A. \& Eichinger, S. (2012) Hematocrit and the risk of recurrent venous thrombosis: a prospective cohort study. PLoS One, 7 , e38705.

Gagnon, D.R., Zhang, T.J., Brand, F.N. \& Kannel, W.B. (1994) Hematocrit and the risk of cardiovascular disease - the Framingham study: a 34-year follow-up. Am. Heart J., 127, 674-682.

Gotoh, S., Hata, J., Ninomiya, T., Hirakawa, Y., Nagata, M., Mukai, N., Fukuhara, M., Ikeda, F., Ago, T., Kitazono, T. \& Kiyohara, Y. (2015) Hematocrit and the risk of cardiovascular disease in a Japanese community: the Hisayama Study. Atherosclerosis, 242, 199-204.

Guyton, A.C. \& Richardson, T.Q. (1961) Effect of hematocrit on venous return. Circ. Res., 9, 157-164.

Harrison, M.J., Pollock, S.S. \& Weisblatt, E. (1984) Haematocrit and platelet aggregation. Lancet, 2, 991-992.

Hellem, A.J., Borchgrevink, C.F. \& Ames, S.B. (1961) The role of red cells in haemostasis: the relation between haematocrit, bleeding time and platelet adhesiveness. Br. J. Haematol., 7, 42-50.

Irie, F., Iso, H., Sairenchi, T., Fukasawa, N., Yamagishi, K., Ikehara, S., Kanashiki, M., Saito, Y., Ota, H. \& Nose, T. (2006) The relationships of proteinuria, serum creatinine, glomerular filtration rate with cardiovascular disease mortality in Japanese general population. Kidney Int., 69, 1264-1271.

Kannel, W.B., Hjortland, M.C., McNamara, P.M. \& Gordon, T. (1976) Menopause and risk of cardiovascular disease: the 
Framingham study. Ann. Intern. Med., 85, 447-452.

Keith, N.M., Wagener, H.P. \& Barker, N.W. (1974) Some different types of essential hypertension: their course and prognosis. Am. J. Med. Sci., 268, 336-345.

Kiyohara, Y., Ueda, K., Hasuo, Y., Fujii, I., Yanai, T., Wada, J., Kawano, H., Shikata, T., Omae, T. \& Fujishima, M. (1986) Hematocrit as a risk factor of cerebral infarction: long-term prospective population survey in a Japanese rural community. Stroke, 17, 687-692.

Konishi, M., Iso, H., Baba, S., Terao, A., Iida, M., Shimamoto, T. \& Komachi, Y. (1992) Pathologic characteristics of stroke and myocardial infarction in Japan: Akita Pathology Study. $J$. Epidemiol., 2, S137-S147.

Kubota, K., Sakurai, T., Tamura, J. \& Shirakura, T. (1987) Is the circadian change in hematocrit and blood viscosity a factor triggering cerebral and myocardial infarction? Stroke, 18, 812-813.

Kunnas, T., Solakivi, T., Huuskonen, K., Kalela, A., Renko, J. \& Nikkari, S.T. (2009) Hematocrit and the risk of coronary heart disease mortality in the TAMRISK study, a 28-year follow-up. Prev. Med., 49, 45-47.

Lowe, G.D. (1998) Etiopathogenesis of cardiovascular disease: hemostasis, thrombosis, and vascular medicine. Ann. Periodontol., 3, 121-126.

Lowe, G.D., Drummond, M.M., Lorimer, A.R., Hutton, I., Forbes, C.D., Prentice, C.R. \& Barbenel, J.C. (1980) Relation between extent of coronary artery disease and blood viscosity. Br. Med. J., 280, 673-674.

Lowe, G.D. \& Forbes, C.D. (1985) Platelet aggregation, haematocrit, and fibrinogen. Lancet, 1, 395-396.

Lowe, G.D., Robertson, J., Dunn, F.G., Lorimer, A.R. \& Forbes, C.D. (1985) Haematocrit in patients attending a hypertension clinic. Scott. Med. J., 30, 168-172.

Lowe, G.D., Rumley, A., Whincup, P.H. \& Danesh, J. (2002) Hemostatic and rheological variables and risk of cardiovascular disease. Semin. Vasc. Med., 2, 429-439.

Mendelsohn, M.E. \& Karas, R.H. (1999) The protective effects of estrogen on the cardiovascular system. N. Engl. J. Med., 340, 1801-1811.

Ministry of Health, Labour and Welfare (2016) Handbook of Health and Welfare Statistics 2015. Health, Labour and Welfare Statistics Association, Tokyo.

Mitsuyama, Y., Thompson, L.R., Hayashi, T., Lee, K.K., Keehn, R.J., Resch, J.A. \& Steer, A. (1979) Autopsy study of cerebrovascular disease in Japanese men who lived in Hiroshima, Japan, and Honolulu, Hawaii. Stroke, 10, 389-395.

Nagai, K., Sairenchi, T., Irie, F., Watanabe, H., Ota, H. \& Yamagata, K. (2016) Relationship between estimated glomerular filtration rate and cardiovascular mortality in a Japanese cohort with long-term follow-up. PLoS One, 11, e0156792.

Nicolaides, A.N., Horbourne, T., Bowers, R., Kidner, P.H. \& Besterman, E.M. (1977) Blood viscosity, red-cell flexibility, haematocrit, and plasma-fibrinogen in patients with angina. Lancet, 2, 943-945.

OECD (2017) Health at a Glance 2017. OECD Publishing, Paris.

Pocock, S.J., Ashby, D., Shaper, A.G., Walker, M. \& Broughton, P.M. (1989) Diurnal variations in serum biochemical and haematological measurements. J. Clin. Pathol., 42, 172-179.

Pristipino, C., Beltrame, J.F., Finocchiaro, M.L., Hattori, R., Fujita, M., Mongiardo, R., Cianflone, D., Sanna, T., Sasayama, S. \& Maseri, A. (2000) Major racial differences in coronary constrictor response between japanese and caucasians with recent myocardial infarction. Circulation, 101, 1102-1108.

Sairenchi, T., Iso, H., Yamagishi, K., Irie, F., Okubo, Y., Gunji, J., Muto, T. \& Ota, H. (2011) Mild retinopathy is a risk factor for cardiovascular mortality in Japanese with and without hypertension: the Ibaraki Prefectural Health Study. Circulation, 124, 2502-2511.

Sennels, H.P., Jorgensen, H.L., Hansen, A.L., Goetze, J.P. \& Fahrenkrug, J. (2011) Diurnal variation of hematology parameters in healthy young males: the Bispebjerg study of diurnal variations. Scand. J. Clin. Lab. Invest., 71, 532-541.

Skafar, D.F., Xu, R., Morales, J., Ram, J. \& Sowers, J.R. (1997) Clinical review 91: Female sex hormones and cardiovascular disease in women. J. Clin. Endocrinol. Metab., 82, 39133918.

Sorlie, P.D., Garcia-Palmieri, M.R., Costas, R. Jr. \& Havlik, R.J. (1981) Hematocrit and risk of coronary heart disease: the Puerto Rico Health Program. Am. Heart J., 101, 456-461.

Thomas, D.J., Marshall, J., Russell, R.W., Wetherley-Mein, G., du Boulay, G.H., Pearson, T.C., Symon, L. \& Zilkha, E. (1977) Effect of haematocrit on cerebral blood-flow in man. Lancet, 2, 941-943.

Tohgi, H., Yamanouchi, H., Murakami, M. \& Kameyama, M. (1978) Importance of the hematocrit as a risk factor in cerebral infarction. Stroke, 9, 369-374.

Touitou, Y., Touitou, C., Bogdan, A., Reinberg, A., Auzeby, A., Beck, H. \& Guillet, P. (1986) Differences between young and elderly subjects in seasonal and circadian variations of total plasma proteins and blood volume as reflected by hemoglobin, hematocrit, and erythrocyte counts. Clin. Chem., 32, 801-804.

Vazquez, B.Y., Martini, J., Tsai, A.G., Johnson, P.C., Cabrales, P. \& Intaglietta, M. (2010) The variability of blood pressure due to small changes of hematocrit. Am. J. Physiol. Heart Circ. Physiol., 299, H863-867.

World Health Organization (2014) Noncommunicable diseases country profiles 2014. WHO, Geneva. 